\title{
Orchard Growth and Fruiting of Micropropagated Apple Trees
}

\author{
Richard H. Zimmerman \\ U.S. Department of Agriculture, Agricultural Research Service, Fruit Laboratory, Beltsville, \\ MD 20705 \\ Stephen S. Miller \\ U.S. Department of Agriculture, Agricultural Research Service, Appalachian Fruit Research \\ Station, Kearneysville, WV 25430
}

Additional index words. Malus domestics, micropropagation, in vitro culture, rootstock, cultural practices, flowering, fruit set, nematodes, Xiphinema spp.

\begin{abstract}
Four apple (Malus domestics Borkh.) cultivars, Northern Spy; Ozark Gold, Stayman, and Rome Beauty, were tissue cultured on their own roots (TC) or were budded on seedling, MM.106, or M.26 rootstock. All four cultivars were planted at Beltsville, Md., and 'Ozark Gold' and 'Stayman' were planted at Kearneysville, W. Va. TC trees produced more vegetative growth than trees budded on MM.106 and M.26 at both locations, but TC trees differed little in size from those budded on seedling rootstock. Flowering was delayed on TC and seedling rootstock trees relative to those on MM.106 or M.26 rootstock. Fruit yields in general were low but were higher for the trees on clonal rootstock than the TC or seedling rootstock trees, especially at Beltsville. The limited vegetative growth and poor fruit yield of trees on M.26 and MM.106 at Beltsville may have been due to significant infestation by plant parasitic nematodes at this site. TC trees seemed to have been less affected by the nematodes, probably because of their greater vigor and more extensive root systems. All trees at Kearneysville were more vigorous than comparable ones at Beltsville.
\end{abstract}

Apple cultivars can be micropropagated (Hutchinson and Zimmerman, 1987; Jones et al., 1979; Zimmerman, 1984; Zimmerman and Broome, 1980, 1981) to produce self-rooted trees readily. However, commercial use of self-rooted TC trees should be considered only after long-term field evaluations have been completed. Such evaluations are currently underway in Italy (Cobianchi et al., 1988; Rosati and Gaggioli, 1987, 1989), in the United Kingdom (Webster et al., 1985), in France (J.C. Navatel, personal communication), in the United States (Larsen et al., 1989; Zimmerman and Miller, 1985, 1986; Zimmerman and Steffens, 1989), and in Canada. Several factors other than propagation method, particularly cultural practices used during the first few years, have been shown to affect the results obtained with micropropagated trees (Zimmerman, 1986). A comparison of the foliar nutrient status of the TC and budded trees used in this experiment has been reported (Korcak et al., 1988).

This experiment was the first established in the United States to compare the performance of TC trees with trees budded on different rootstock and growing on sites typical of those used in the apple industry in the eastern United States.

\section{Materials and Methods}

'Ozark Gold', 'Stayman', 'Northern Spy', and 'Rome Beauty' apples were micropropagated as described by Zimmerman and Broome $(1980,1981)$, and the trees were planted in a nursery at Beltsville in late May 1980. In Spring 1981, all lateral branches were removed and the main shoot was headed at a height of $\approx 70$ to $80 \mathrm{~cm}$. The trees were dug from the nursery in Dec. 1981 and placed in a common storage for the winter. Budwood was collected from the same orchard-grown trees from which explants were taken for micropropagation. This budwood was used to propagate trees on seedling, MM. 106, and M.26 root-

Received for publication 25 May 1990. The cost of publishing this paper was defrayed in part by the payment of page charges. Under postal regulations, this paper therefore must be hereby marked advertisement solely to indicate this fact. stocks at a commercial nursery in Summer 1980. The budded trees were also dug in Dec. 1981 and put in storage.

Trees of 'Ozark Gold', 'Stayman', and 'Northern Spy' were planted in Apr. 1982 at Beltsville and Kearneysville, using the same strip split-plot design at each site. Rootstock were the main plots and cultivars the subplots; four replications with four-tree experimental units were used at each site. Due to logistical problems, data were not collected on trees of 'Northern Spy' at Kearneysville. At Beltsville, trees of 'Rome Beauty' were planted adjacent to the above planting in a Latin-square design with four trees per plot and four replications. Trees were planted in rows $4.9 \mathrm{~m}$ apart with a between-tree spacing of 3.0 $\mathrm{m}$ for M.26, $3.7 \mathrm{~m}$ for MM.106, and $4.3 \mathrm{~m}$ for seedling and TC. After planting, graft unions for budded trees were at or slightly above ground at Keameysville and $\approx 10 \mathrm{~cm}$ above ground level at Beltsville. The TC trees were taller and had more branches than budded trees at time of planting, so all lateral branches were removed from all trees and the main shoot was headed to $\approx 1 \mathrm{~m}$ to make the trees nearly equal in size. Trees were trained to a modified central leader system. The trees were not staked.

The Beltsville site, a Beltsville fine sandy loam (Typic Fragiudult, fine-loamy, mixed, mesic), had been used continuously for apple and peach orchards for at least 30 years before this orchard was planted. At Kearneysville, the trees were planted on a site of Hagerstown-Frederick cherty silt loam soil (Typic Hapludalf, fine, mixed, mesic) that had been used for corn the preceding 10 years and for pasture before that.

Cultural practices differed at the two sites. Trees planted at Beltsville were broadcast-fertilized in 1982 with $\left(\mathrm{kg} \cdot \mathrm{ha}^{-1}\right) 336$ $10 \mathrm{~N}-4.4 \mathrm{P}-8.3 \mathrm{~K}$ plus $22.4 \mathrm{Mg}$ and annually from 1983 through 1986 only with urea (37.8 actual N). At Kearneysville, fertilizer applications were $\left(\mathrm{kg} \cdot \mathrm{ha}^{-1}\right) 45$ actual $\mathrm{N}$ from $\mathrm{Ca}\left(\mathrm{NO}_{3}\right)_{2}$ in 1982 , 90 actual $\mathrm{N}$ from $\mathrm{Ca}\left(\mathrm{NO}_{3}\right)_{2}$ plus $67.2 \mathrm{Mg}$ from $\mathrm{MgSO}_{4}$ in 1983 and 39 actual $\mathrm{N}$ from urea each year for 1984-86. An in-row weed-free strip $1.2 \mathrm{~m}$ wide at Beltsville and $2.4 \mathrm{~m}$ wide at

Abbreviations: TC, tissue cultured; TCSA, trunk cross-sectional area. 
Kearneysville was maintained using paraquat. Trees at Beltsville were sprinkler-irrigated as needed in 1982, 1983, and early 1984. In 1984, a drip irrigation system was installed and used for the remainder of the experiment. Trees at Kearneysville were irrigated at planting only.

Data collected on each tree included trunk diameter (Beltsville) or circumference (Kearneysville) $30 \mathrm{~cm}$ above the ground for TC and $30 \mathrm{~cm}$ above the graft union for budded trees, taken in the fall each year, plus at time of planting for the Beltsville trees; length of all first and second order shoots, and angle between each branch and the trunk in 1982; length of five (1983) or 10 (1984) lateral shoots; height, spread in-row and acrossrow in 1984, 1985, and 1986. TCSA was calculated for each tree. Trees were evaluated for flowering by use of a rating scale (Beltsville; Zimmerman, 1977) or by counting all flower clusters (Kearneysville); all fruit were harvested and weighed in 1985 and 1986. When the experiment was terminated in Spring 1987, two representative trees in each treatment at each location of 'Stayman' and 'Ozark Gold' (three trees per treatment of 'Ozark Gold' at Beltsville) were destructively harvested. The trees were cut down before budbreak and cut into small sections to aid drying. The root system was sampled using a Vermeer tree spade (Vermeer Manufacturing, Pella, Iowa) to dig the roots surrounding the crown. These were contained in a frustum of soil $95 \mathrm{~cm}$ in diameter at the soil surface and $20 \mathrm{~cm}$ in diameter at a depth of $75 \mathrm{~cm}$, a volume of $0.22 \mathrm{~m}^{3}$. Soil was removed from the crown and roots by shaking and then washing. The number of primary roots originating from the trunk and any secondary roots on these primaries were counted, and the diameter of each root was measured $20 \mathrm{~cm}$ from the trunk. Crosssectional areas were calculated for each root and these were summed for each tree. Dry weights for the tops and the roots plus crown were determined after preliminary drying in a greenhouse followed by drying to a constant weight in an oven at 60C.

Identification and counts (number per $250 \mathrm{ml}$ solid soil sample) of nematodes in soil samples collected at the Beltsville site were done by the Nematode Diagnostic Laboratory, Univ. of Maryland, College Park.

Data were analyzed using the General Linear Model procedure of SAS (SAS Institute, Inc., 1982). Covariate analysis of all variables, using TCSA at time of planting as the covariate, was performed each year. Since the covariate was significant only for TCSA and only for the first year's data, no adjustments were made to any data presented here. In the split-plot experiments, data were analyzed both for all cultivars combined and for each cultivar separately. Since the results were essentially the same by either method and since difference in performance among rootstock was the main objective of the study, only the latter analyses are presented.

Table 1. Growth of self-rooted TC apple trees compared with budded trees. ${ }^{\mathbf{z}}$

\begin{tabular}{|c|c|c|c|c|c|c|c|c|c|}
\hline \multirow[b]{2}{*}{ Rootstock } & \multirow{2}{*}{$\begin{array}{c}\text { No. of } \\
\text { shoots, } \\
1982 \\
\end{array}$} & \multirow{2}{*}{$\begin{array}{c}\text { Total } \\
\text { shoot } \\
\text { length, } 1982 \\
(\mathrm{~m})\end{array}$} & \multirow{2}{*}{$\begin{array}{c}\text { Branch } \\
\text { angle, } \\
1982 \\
\left({ }^{\circ}\right) \\
\end{array}$} & \multicolumn{2}{|c|}{$\begin{array}{l}\text { Shoot length } \\
\text { (cm) }\end{array}$} & \multirow{2}{*}{$\begin{array}{c}\mathrm{Ht} \\
(\mathrm{m}) \\
1984\end{array}$} & \multicolumn{3}{|c|}{$\begin{array}{l}\text { Spread in row } \\
(\mathrm{m})\end{array}$} \\
\hline & & & & 1983 & 1984 & & 1984 & 1985 & 1986 \\
\hline \multicolumn{10}{|c|}{ Ozark Gold-Beltsville } \\
\hline $\mathrm{TC}$ & $15 \mathrm{~b}$ & $4.8 \mathrm{a}$ & $81 \mathrm{a}$ & $90 \mathrm{a}$ & $80 \mathrm{a}$ & $2.6 \mathrm{a}$ & $1.7 \mathrm{a}$ & $2.6 \mathrm{a}$ & $3.0 \mathrm{a}$ \\
\hline Seedling & $16 a b$ & $2.2 \mathrm{~b}$ & $74 \mathrm{a}$ & $81 \mathrm{ab}$ & $76 \mathrm{a}$ & $2.6 \mathrm{a}$ & $1.4 \mathrm{~b}$ & $2.3 \mathrm{ab}$ & $2.8 \mathrm{a}$ \\
\hline MM.106 & $17 \mathrm{a}$ & $2.6 \mathrm{~b}$ & $79 a$ & $64 \mathrm{c}$ & $59 \mathrm{~b}$ & $2.3 \mathrm{a}$ & $1.3 \mathrm{~b}$ & $2.0 \mathrm{bc}$ & $2.2 \mathrm{~b}$ \\
\hline M.26 & $13 \mathrm{c}$ & $2.2 \mathrm{~b}$ & 72 a & $71 \mathrm{bc}$ & $54 \mathrm{~b}$ & $2.3 \mathrm{a}$ & $1.3 \mathrm{~b}$ & $1.8 \mathrm{c}$ & $2.2 \mathrm{~b}$ \\
\hline \multicolumn{10}{|c|}{ Ozark Gold-Kearneysville } \\
\hline $\mathrm{TC}$ & 11 a & $5.7 \mathrm{a}$ & 78 a & 74 a & $73 \mathrm{a}$ & $3.2 \mathrm{a}$ & $2.4 \mathrm{a}$ & $3.7 \mathrm{a}$ & $4.6 \mathrm{a}$ \\
\hline Seedling & $5 b$ & $2.9 \mathrm{c}$ & $55 \mathrm{~b}$ & $72 a$ & $74 \mathrm{a}$ & $2.9 \mathrm{a}$ & $1.8 \mathrm{~b}$ & $3.1 \mathrm{~b}$ & $4.3 \mathrm{ab}$ \\
\hline MM.106 & $7 b$ & $4.5 \mathrm{~b}$ & $84 a$ & $73 \mathrm{a}$ & $71 \mathrm{a}$ & $3.2 \mathrm{a}$ & $1.9 \mathrm{~b}$ & $3.3 \mathrm{~b}$ & $4.0 \mathrm{~b}$ \\
\hline M.26 & $7 \mathrm{~b}$ & $4.0 \mathrm{~b}$ & 80 a & $78 \mathrm{a}$ & 67 a & $3.0 \mathrm{a}$ & $1.9 \mathrm{~b}$ & $3.0 \mathrm{~b}$ & $3.9 \mathrm{~b}$ \\
\hline \multicolumn{10}{|c|}{ Stayman-Beltsville } \\
\hline $\mathrm{TC}$ & $14 \mathrm{a}$ & $4.0 \mathrm{a}$ & $75 \mathrm{a}$ & 92 a & 83 a & $2.8 \mathrm{a}$ & $1.8 \mathrm{a}$ & $2.9 \mathrm{a}$ & $3.4 \mathrm{a}$ \\
\hline Seedling & $16 \mathrm{a}$ & $1.6 \mathrm{~b}$ & $70 \mathrm{a}$ & $68 \mathrm{~b}$ & $81 \mathrm{a}$ & $2.6 \mathrm{a}$ & $1.4 \mathrm{~b}$ & $2.4 \mathrm{~b}$ & $2.7 \mathrm{~b}$ \\
\hline MM.106 & 15 a & $1.3 \mathrm{~b}$ & $74 \mathrm{a}$ & $50 \mathrm{c}$ & $53 \mathrm{~b}$ & $2.0 \mathrm{~b}$ & $1.0 \mathrm{c}$ & $1.8 \mathrm{c}$ & $1.9 \mathrm{c}$ \\
\hline M.26 & 15 a & $1.3 \mathrm{~b}$ & $67 \mathrm{a}$ & $47 \mathrm{c}$ & $40 \mathrm{c}$ & $1.9 \mathrm{~b}$ & $1.0 \mathrm{c}$ & $1.5 \mathrm{~d}$ & $1.9 \mathrm{c}$ \\
\hline \multicolumn{10}{|c|}{ Stayman-Kearneysville } \\
\hline TC & $10 \mathrm{a}$ & $6.6 \mathrm{a}$ & $54 \mathrm{ab}$ & 82 a & 85 a & $3.4 \mathrm{a}$ & $1.8 \mathrm{a}$ & $3.1 \mathrm{a}$ & $4.3 \mathrm{a}$ \\
\hline Seedling & $5 \mathrm{~b}$ & $4.4 \mathrm{~b}$ & $52 \mathrm{~b}$ & 89 a & $88 \mathrm{a}$ & $3.2 \mathrm{a}$ & $1.7 \mathrm{a}$ & $2.7 \mathrm{ab}$ & $3.7 \mathrm{~b}$ \\
\hline MM.106 & $5 \mathrm{~b}$ & $4.3 \mathrm{~b}$ & $50 \mathrm{a}$ & $80 \mathrm{a}$ & $80 \mathrm{a}$ & $3.1 \mathrm{a}$ & $1.9 \mathrm{a}$ & $2.9 \mathrm{ab}$ & $4.0 \mathrm{ab}$ \\
\hline M.26 & $4 \mathrm{~b}$ & $3.3 \mathrm{~b}$ & $57 \mathrm{ab}$ & $76 \mathrm{a}$ & $51 \mathrm{~b}$ & $2.6 \mathrm{~b}$ & $1.5 \mathrm{a}$ & $2.4 \mathrm{~b}$ & $3.4 \mathrm{~b}$ \\
\hline \multicolumn{10}{|c|}{ Northern Spy-Beltsville } \\
\hline $\mathrm{TC}$ & $13 \mathrm{a}$ & $3.9 \mathrm{a}$ & 59 a & 109 a & $96 \mathrm{a}$ & $2.9 \mathrm{a}$ & $1.2 \mathrm{a}$ & $1.8 \mathrm{a}$ & $2.1 \mathrm{~b}$ \\
\hline Seedling & $10 \mathrm{~b}$ & $2.2 \mathrm{~b}$ & $54 \mathrm{a}$ & 103 a & $101 \mathrm{a}$ & $2.8 \mathrm{ab}$ & $1.0 \mathrm{~b}$ & $1.7 \mathrm{a}$ & $1.8 \mathrm{~b}$ \\
\hline MM.106 & $9 \mathrm{bc}$ & $2.0 \mathrm{~b}$ & $56 \mathrm{a}$ & $89 \mathrm{~b}$ & $69 \mathrm{~b}$ & $2.6 \mathrm{bc}$ & $0.9 \mathrm{~b}$ & $1.5 \mathrm{a}$ & $1.8 \mathrm{~b}$ \\
\hline M.26 & 7. c & $1.5 \mathrm{~b}$ & $48 \mathrm{~b}$ & $87 \mathrm{~b}$ & $64 \mathrm{~b}$ & $2.4 \mathrm{c}$ & $1.1 \mathrm{a}$ & $1.8 \mathrm{a}$ & $2.6 \mathrm{a}$ \\
\hline \multicolumn{10}{|c|}{ Rome Beauty-Beltsville } \\
\hline $\mathrm{TC}$ & $14 \mathrm{a}$ & $4.8 \mathrm{a}$ & $57 \mathrm{a}$ & 86 a & $79 \mathrm{ab}$ & $2.4 \mathrm{a}$ & $1.2 \mathrm{a}$ & $2.1 \mathrm{a}$ & $2.4 \mathrm{a}$ \\
\hline Seedling & $12 \mathrm{a}$ & $2.7 \mathrm{ab}$ & $57 \mathrm{a}$ & $69 a$ & $82 \mathrm{a}$ & $2.4 \mathrm{a}$ & $1.1 \mathrm{a}$ & $1.9 \mathrm{a}$ & $2.3 \mathrm{ab}$ \\
\hline MM.106 & $12 \mathrm{a}$ & $2.8 \mathrm{~b}$ & $59 \mathrm{a}$ & $62 \mathrm{a}$ & $61 \mathrm{bc}$ & $2.2 \mathrm{a}$ & $1.1 \mathrm{a}$ & $1.9 \mathrm{a}$ & $2.3 \mathrm{ab}$ \\
\hline M.26 & $13 \mathrm{a}$ & $2.2 \mathrm{~b}$ & $57 \mathrm{a}$ & $65 \mathrm{a}$ & $52 \mathrm{c}$ & $2.1 \mathrm{a}$ & $1.1 \mathrm{a}$ & $1.6 \mathrm{~b}$ & $2.0 \mathrm{~b}$ \\
\hline
\end{tabular}

${ }^{z}$ Mean separation in columns for each cultivar-location by Duncan's multiple range test, $P=0.05$. 


\section{Results}

Orchard establishment. All trees planted at Beltsville survived and grew. No loss of TC trees occurred until the fifth growing season, when one tree of 'Northern Spy' died. By the end of the experiment, six trees on M.26 and one on MM. 106 were lost, all but one due to breakage at the graft union following a wind storm in Sept. 1985. Six trees died in the year of planting at Kearneysville: two 'Ozark Gold' on seedling and one on M.26, and two 'Stayman' on seedling and one TC. In the second growing season, three trees of 'Ozark Gold' died, one each on MM.106 and M.26 and one TC, and one tree of 'Stayman' on M.26 died. Five more trees of 'Stayman' on M.26 died in 1985. The majority of trees budded on M.26 that died at Kearneysville during the study were diagnosed as having fire blight (incited by Erwinia amylovora).

Vegetative growth. During the first growing season (1982), TC trees usually had more shoots than, or as many as, the budded trees and produced more shoot growth than budded trees at both locations (Table 1). Branch angle was as great for TC trees as for any of the budded trees. Trees at Kearneysville generally produced more shoot growth than those at Beltsville, especially the budded trees.

Rootstock had no effect on tree size as measured by TCSA at time of orchard planting at Beltsville (data not shown) except for 'Rome Beauty'. For this cultivar, trees budded on MM.106 $\left(0.93 \mathrm{~cm}\right.$ ' TCSA) and M.26 (1.1 $\mathrm{cm}^{2}$ TCSA $)$ were significantly larger than those on seedling rootstock $\left(0.70 \mathrm{~cm}^{2}\right.$ TCSA); TC trees $\left(0.78 \mathrm{~cm}^{2}\right.$ TCSA) did not differ from those on seedling or MM.106.

By the end of the first growing season (1982), TC trees of 'Northern Spy' (Fig. 1E) and 'Stayman' (Fig. 1 C, D) had significantly larger TCSA than any budded trees; 'Ozark Gold' on seedling at Kearneysville was significantly smaller than those on all other rootstock (Fig. 1B), while no differences among
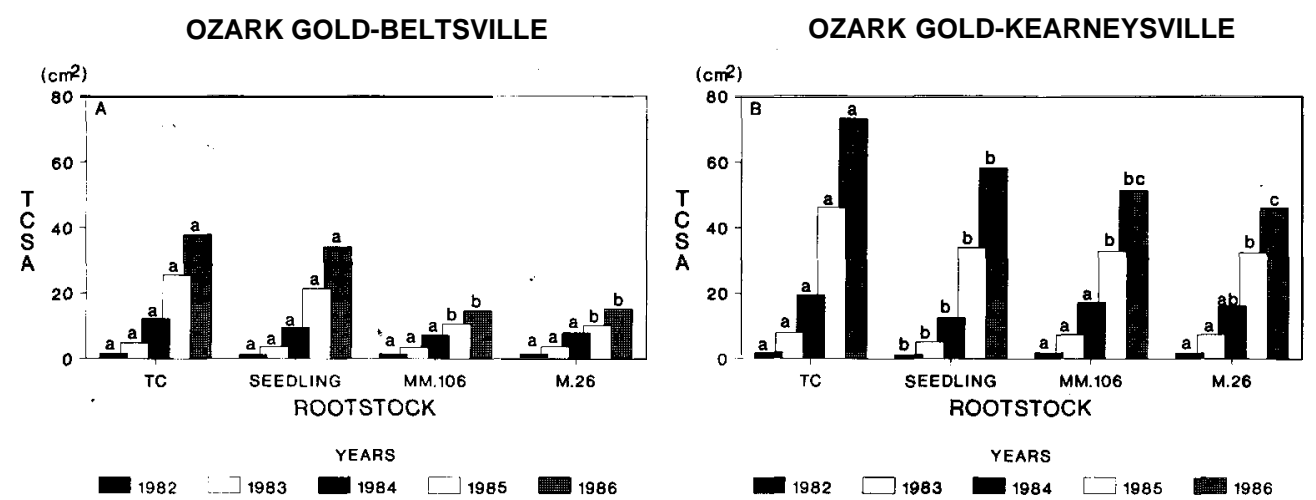

STAYMAN-BELTSVILLE
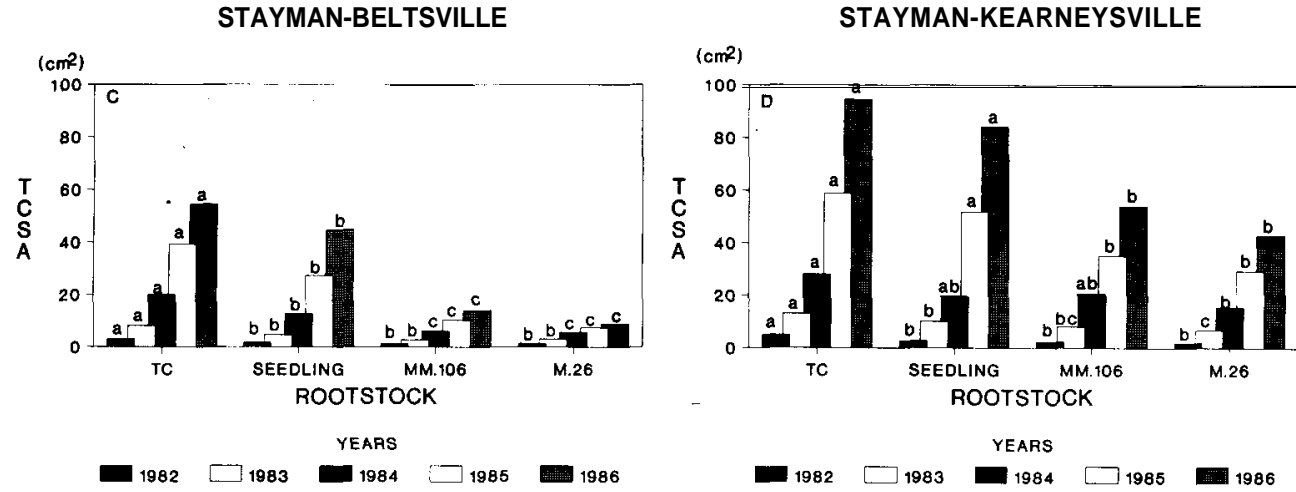

NORTHERN SPY-BELTSVILLE
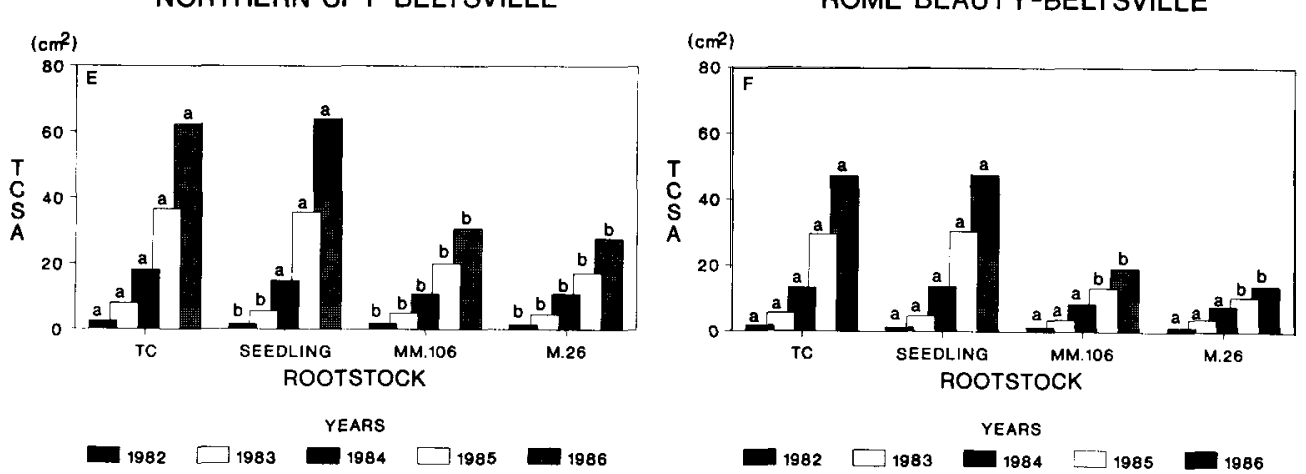

Fig. 1. TCSA of (A) 'Ozark Gold' at Beltsville, (B) 'Ozark Gold' at Kearneysville, (C) 'Stayman' at Beltsville, (D) 'Stayman' at Kearneysville, (E) 'Northern Spy' at Beltsville, and (F) 'Rome Beauty' at Beltsville, grown as self-rooted TC trees or budded on seedling, MM.106, or M.26 rootstock. For a given year for each cultivar-location combination, bars (rootstock) topped by a different letter differ by Duncan's multiple range test, $P=0.05$. 
rootstock existed for 'Ozark Gold' or 'Rome Beauty' at Beltsville (Fig. 1A, F).

After the first growing season, TC trees and those on seedling rootstock at Beltsville were equivalent in vigor and grew more vigorously than trees on MM. 106 or M.26 as measured by TCSA (Fig. 1), shoot length (1983 and 1984), height (1984), and inrow spread (1984-86) (Table 1). Similar results were shown by height and spread across rows (data not shown). Cultivars responded dissimilarly. Those less vigorous ('Ozark Gold' and 'Rome Beauty') did not show differences in TCSA until the end of 1985, after four growing seasons in the orchard. For the more vigorous cultivars (Stayman and Northern Spy), the TC trees had a significantly larger TCSA after the first growing season (1982), and this difference was maintained throughout the experiment. Trees of 'Northern Spy'/seedling were as large as the TC trees after the third growing season (Fig. 1E).

After the first growing season, all trees planted at Kearneysville continued to grow much more vigorously than those at Beltsville. Differences in TCSA among rootstock were not as clear-cut at Kearneysville during the first three growing seasons (1982-84), but, by the end of the 4th year, a pattern of differences similar to that at Beltsville had emerged (Fig. 1B, D). Other measures of vegetative growth generally reflected the differences shown by TCSA (Table 1).

Final tree size. Data for trees harvested at the end of the experiment at Beltsville showed that 'Ozark Gold' and 'Stayman' TC and seedling rootstock trees were significantly larger than those on MM.106 or M.26 as measured by dry weight of the tops and of the roots harvested (Table 2). Data on number of roots and root cross-sectional area were consistent with this observation, although the substantial differences were not always statistically significant, probably because of the limited replication for harvested trees.

Table 2. Final size of self-rooted TC apple trees compared with budded trees after 5 years in the orchard.

\begin{tabular}{|c|c|c|c|c|}
\hline \multirow[b]{2}{*}{ Rootstock } & \multicolumn{2}{|c|}{$\begin{array}{c}\text { Dry wt } \\
(\mathrm{kg})\end{array}$} & \multirow{2}{*}{$\begin{array}{l}\text { Total } \\
\text { roots } \\
\text { (no.) }\end{array}$} & \multirow{2}{*}{$\begin{array}{c}\text { Root } \\
\text { cross- } \\
\text { sectional } \\
\text { area } \\
\left(\mathrm{cm}^{2}\right)\end{array}$} \\
\hline & Top & Root & & \\
\hline \multicolumn{5}{|c|}{ Ozark Gold-Beltsville } \\
\hline TC & $8.9 \mathrm{a}$ & $2.5 \mathrm{a}$ & $37.7 \mathrm{a}$ & $54 \mathrm{~b}$ \\
\hline Seedling & $9.1 \mathrm{a}$ & $3.1 \mathrm{a}$ & $28.3 \mathrm{ab}$ & 95 a \\
\hline MM.106 & $2.3 \mathrm{~b}$ & $1.2 \mathrm{~b}$ & $14.3 \mathrm{~b}$ & $32 \mathrm{~b}$ \\
\hline M.26 & $2.3 \mathrm{~b}$ & $0.9 \mathrm{~b}$ & $21.7 \mathrm{ab}$ & $19 \mathrm{~b}$ \\
\hline \multicolumn{5}{|c|}{ Ozark Gold-Kearneysville } \\
\hline $\mathrm{TC}$ & $16.7 \mathrm{a}$ & $4.3 \mathrm{a}$ & $36.5 \mathrm{a}$ & $104 a b$ \\
\hline Seedling & $15.1 \mathrm{a}$ & $5.5 \mathrm{a}$ & $42.5 \mathrm{a}$ & 159 a \\
\hline MM.106 & $9.8 \mathrm{a}$ & $3.0 \mathrm{a}$ & $33.0 \mathrm{ab}$ & $64 \mathrm{~b}$ \\
\hline M.26 & $16.0 \mathrm{a}$ & $5.7 \mathrm{a}$ & $21.5 \mathrm{~b}$ & $136 \mathrm{a}$ \\
\hline \multicolumn{5}{|c|}{ Stayman-Beltsville } \\
\hline $\mathrm{TC}$ & $10.8 \mathrm{a}$ & $4.3 \mathrm{a}$ & $41.0 \mathrm{a}$ & 109 a \\
\hline Seedling & $10.4 \mathrm{a}$ & $3.0 \mathrm{ab}$ & $29.5 \mathrm{ab}$ & $108 \mathrm{a}$ \\
\hline MM.106 & $1.5 \mathrm{~b}$ & $0.7 \mathrm{~b}$ & $11.0 \mathrm{~b}$ & 19 a \\
\hline M.26 & $1.3 \mathrm{~b}$ & $0.5 \mathrm{~b}$ & $20.0 \mathrm{~b}$ & $9.6 \mathrm{a}$ \\
\hline \multicolumn{5}{|c|}{ Stayman-Kearneysville } \\
\hline $\mathrm{TC}$ & $30.3 \mathrm{a}$ & $6.6 \mathrm{a}$ & $26.5 \mathrm{a}$ & 139 a \\
\hline Seedling & $19.2 \mathrm{ab}$ & $4.9 \mathrm{ab}$ & $51.0 \mathrm{a}$ & $93 a b$ \\
\hline MM.106 & $10.0 \mathrm{~b}$ & $2.5 \mathrm{~b}$ & $28.0 \mathrm{a}$ & $55 \mathrm{~b}$ \\
\hline M.26 & $17.9 \mathrm{~b}$ & $4.0 \mathrm{~b}$ & $26.5 \mathrm{a}$ & $110 \mathrm{a}$ \\
\hline
\end{tabular}

${ }^{7}$ Mean separation in columns for each cultivar-location by Duncan's multiple range test, $P=0.05$.
For trees grown at Kearneysville, the data on the harvested trees were not as clear, reflecting the more vigorous growth of trees budded on clonal rootstock at that site (Table 2). For 'Ozark Gold', none of the weight measurements were statistically different. Trees budded on M.26 had the fewest roots and those on seedling had the most. However, root size was smallest on MM.106 and greatest on seedling and M.26. Fresh (data not shown) and dry weights for 'Stayman' were highest for TC and seedling rootstock trees, as was true also at Beltsville. The data for roots showed that the seedling rootstock tended to produce more but smaller roots and, again, MM. 106 had the smallest root system.

Flower and fruit production. Scattered flowering occurred on trees budded on MM.106 and M.26 the year of planting (1982) and on all budded trees the following year. Flowering of TC trees did not begin until the start of the third growing season (1984) and increased rapidly thereafter for 'Stayman' and 'Rome Beauty' (Table 3). Substantial flowering of 'Ozark Gold' trees on TC or seedling rootstock did not occur until the start of the fifth growing season at Beltsville, by which time the trees on MM. 106 and M.26 had relatively few flowers. Flowering of 'Northern Spy' was always light, although it was better on M.26 and MM.106 than for TC or trees or seedling rootstock, especially in the 5 th year.

No fruit was picked at Beltsville in 1984 although a few scattered fruit set on trees budded on MM.106 and M.26. In 1985, yields of 'Ozark Gold' and 'Rome Beauty' at Beltsville were higher on M.26 and MM.106 than on seedling or TC, but in 1986, yields were higher for TC and seedling rootstock trees of 'Ozark Gold' with no difference for 'Rome Beauty' (Table 3). TC trees of 'Stayman' yielded more both years at Beltsville than any of the others. However, yields for all trees at Beltsville were low.

At Kearneysville in 1984, an average of 15 to 20 fruit were picked from 'Ozark Gold' trees on seedling, MM.106, and M.26 rootstock, while 'Stayman' trees on MM. 106 produced 25 to 30 fruit and those on other roots produced two to five fruit. 'Ozark Gold' on MM.106 and M.26 had higher yields than on seedling or TC in both 1985 and 1986 as did 'Stayman' in 1986 (Table 3). 'Stayman' at Kearneysville in 1985 had higher yields on MM.106 and TC than on seedling and M.26.

Cumulative yield efficiency (kilograms of fruit per square centimeter TCSA) was significantly higher for M.26 and MM. 106 than for seedling or TC for all cultivars except for 'Stayman' at Beltsville, where no differences existed (Table 3).

Nematode populations. After a preliminary soil sample taken at the Beltsville site in early Sept. 1985 showed a high count of Xiphinema spp., more extensive sampling (20 samples for the 0.8-ha site) was conducted in late Sept. 1985 and early Oct. 1986. Nematode populations high enough $(395 \pm 379$ per 250 $\mathrm{ml}$ soil) to affect plant growth were found in both years. Most prevalent were Xiphinema spp., followed by Pratylenchus spp., Tylechorhynchus spp., Helicotylenchus spp., and Hoplolaimus Spp.

Nematode populations in the soil at Kearneysville were negligible.

Tree breakage. Following a wind storm at Beltsville in early Sept. 1985, several trees on M.26 ['Ozark Gold' (1), 'Northern Spy' (1), 'Stayman' (1) and 'Rome Beauty' (2)] and one tree on MM.106 ('Northern Spy') broke off cleanly at the graft union. Examination of the graft union showed that symptoms consistent with apple union necrosis and decline, caused by tomato ring spot virus, were present. Trees on these rootstock that did not 
Table 3. Flowering and fruiting behavior for self-rooted TC and budded apple trees. ${ }^{\mathrm{x}}$

\begin{tabular}{|c|c|c|c|c|c|c|c|c|}
\hline \multirow[b]{2}{*}{ Rootstock } & \multicolumn{5}{|c|}{$\begin{array}{c}\text { Flowering rating } \\
\text { (no. flower clusters)y }\end{array}$} & \multicolumn{2}{|c|}{ Fruit yield (kg/tree) } & \multirow{2}{*}{$\begin{array}{c}\text { Yield } \\
\text { efficiency } \\
\left(\mathrm{kg} \cdot \mathrm{cm}^{-2}\right)\end{array}$} \\
\hline & 19 & 84 & 1985 & & 1986 & 1985 & 1986 & \\
\hline $\begin{array}{l}\text { TC } \\
\text { Seedling } \\
\text { MM.106 } \\
\text { M.26 }\end{array}$ & $\begin{array}{l}1.1 \mathrm{~b} \\
1.1 \mathrm{~b} \\
3.1 \mathrm{a} \\
4.0 \mathrm{a}\end{array}$ & $\begin{array}{r}(1) \\
(1) \\
(8) \\
(18)\end{array}$ & $\begin{array}{l}3.1 \mathrm{~b} \\
2.9 \mathrm{~b} \\
6.0 \mathrm{a} \\
6.8 \mathrm{a}\end{array}$ & $\begin{array}{r}O z t \\
(8) \\
(7) \\
(75) \\
(148)\end{array}$ & $\begin{array}{cc}r k \text { Gold-Beltsv } \\
7.4 \mathrm{a} & (250) \\
7.4 \mathrm{a} & (250) \\
5.0 \mathrm{ab} & (38) \\
4.2 \mathrm{~b} & (22)\end{array}$ & $\begin{array}{l}3.0 \mathrm{~b} \\
1.9 \mathrm{~b} \\
7.8 \mathrm{ab} \\
11.8 \mathrm{a}\end{array}$ & $\begin{array}{r}10.9 \mathrm{a} \\
12.3 \mathrm{a} \\
4.4 \mathrm{~b} \\
6.0 \mathrm{~b}\end{array}$ & $\begin{array}{l}0.36 \mathrm{~b} \\
0.42 \mathrm{~b} \\
0.84 \mathrm{a} \\
0.96 \mathrm{a}\end{array}$ \\
\hline $\begin{array}{l}\text { TC } \\
\text { Seedling } \\
\text { MM.106 } \\
\text { M.26 }\end{array}$ & : & $\begin{array}{l}(1) \mathrm{a} \\
(19) \mathrm{a} \\
(54) \mathrm{a} \\
(66) \mathrm{a}\end{array}$ & & Ozarh & $\begin{array}{c}\text { Gold-Keamey } \\
--- \\
--- \\
--- \\
---\end{array}$ & $\begin{array}{l}\text { ille } \\
6.1 \mathrm{~b} \\
4.7 \mathrm{~b} \\
20.2 \mathrm{a} \\
20.2 \mathrm{a}\end{array}$ & $\begin{array}{l}28.4 \mathrm{~b} \\
24.0 \mathrm{~b} \\
44.8 \mathrm{a} \\
40.4 \mathrm{a}\end{array}$ & $\begin{array}{l}0.48 \mathrm{~b} \\
0.49 \mathrm{~b} \\
1.28 \mathrm{a} \\
1.35 \mathrm{a}\end{array}$ \\
\hline $\begin{array}{l}\text { TC } \\
\text { Seedling } \\
\text { MM.106 } \\
\text { M.26 }\end{array}$ & $\begin{array}{l}1.5 \mathrm{~b} \\
2.9 \mathrm{ab} \\
3.7 \mathrm{a} \\
4.0 \mathrm{a}\end{array}$ & $\begin{array}{r}(2) \\
(7) \\
(14) \\
(18)\end{array}$ & $\begin{array}{l}6.7 \mathrm{a} \\
7.0 \mathrm{a} \\
7.1 \mathrm{a} \\
6.8 \mathrm{a}\end{array}$ & $\begin{array}{l}S t \\
(132) \\
(173) \\
(188) \\
(148)\end{array}$ & $\begin{array}{cc}y \text { man-Beltsvil } \\
8.1 \mathrm{a} & (388) \\
7.6 \mathrm{ab} & (290) \\
6.9 \mathrm{bc} & (163) \\
6.1 \mathrm{c} & (80)\end{array}$ & $\begin{array}{l}9.6 \mathrm{a} \\
4.3 \mathrm{ab} \\
4.0 \mathrm{ab} \\
2.2 \mathrm{~b}\end{array}$ & $\begin{array}{l}9.3 \mathrm{a} \\
4.8 \mathrm{~b} \\
1.9 \mathrm{bc} \\
0.8 \mathrm{c}\end{array}$ & $\begin{array}{l}0.35 \mathrm{a} \\
0.21 \mathrm{a} \\
0.43 \mathrm{a} \\
0.31 \mathrm{a}\end{array}$ \\
\hline $\begin{array}{l}\text { TC } \\
\text { Seedling } \\
\text { MM.106 } \\
\text { M.26 }\end{array}$ & & $\begin{array}{r}(6) \mathrm{b} \\
(6) \mathrm{b} \\
(123) \mathrm{a} \\
(97) \mathrm{a}\end{array}$ & - & $\begin{array}{ll} & \text { Stay } \\
-- & \\
\cdots- & \end{array}$ & $\begin{array}{c}\text { nan-Kearneys } \\
--- \\
--- \\
-- \\
---\end{array}$ & $\begin{array}{r}l e \\
10.1 \mathrm{a} \\
4.7 \mathrm{~b} \\
12.0 \mathrm{a} \\
2.1 \mathrm{~b}\end{array}$ & $\begin{array}{r}6.8 \mathrm{~b} \\
3.8 \mathrm{~b} \\
20.6 \mathrm{a} \\
18.5 \mathrm{a}\end{array}$ & $\begin{array}{l}0.18 \mathrm{~b} \\
0.10 \mathrm{~b} \\
0.62 \mathrm{a} \\
0.51 \mathrm{a}\end{array}$ \\
\hline $\begin{array}{l}\text { TC } \\
\text { Seedling } \\
\text { MM.106 } \\
\text { M.26 }\end{array}$ & $\begin{array}{l}1.1 \mathrm{~b} \\
1.0 \mathrm{~b} \\
1.1 \mathrm{~b} \\
1.5 \mathrm{a}\end{array}$ & $\begin{array}{l}(1) \\
(0) \\
(1) \\
(2)\end{array}$ & $\begin{array}{l}1.8 \mathrm{~b} \\
1.1 \mathrm{~b} \\
2.0 \mathrm{~b} \\
4.7 \mathrm{a}\end{array}$ & $\begin{array}{l}\text { Nor } \\
(2) \\
(1) \\
(3) \\
(31)\end{array}$ & \begin{tabular}{lr}
\multicolumn{3}{c}{ Spy-Belts } \\
$4.0 \mathrm{~b}$ & $(18)$ \\
$1.9 \mathrm{c}$ & $(3)$ \\
$5.8 \mathrm{a}$ & $(65)$ \\
$6.0 \mathrm{a}$ & $(75)$
\end{tabular} & $\begin{array}{c}0.04 \mathrm{~b} \\
--- \\
0.3 \mathrm{~b} \\
1.7 \mathrm{a}\end{array}$ & $\begin{array}{l}2.0 \mathrm{bc} \\
0.6 \mathrm{c} \\
5.0 \mathrm{ab} \\
5.8 \mathrm{a}\end{array}$ & $\begin{array}{l}0.03 \mathrm{c} \\
0.01 \mathrm{c} \\
0.18 \mathrm{~b} \\
0.31 \mathrm{a}\end{array}$ \\
\hline $\begin{array}{l}\text { TC } \\
\text { Seedling } \\
\text { MM.106 } \\
\text { M.26 }\end{array}$ & $\begin{array}{l}2.7 \mathrm{~b} \\
3.1 \mathrm{~b} \\
4.6 \mathrm{a} \\
5.0 \mathrm{a}\end{array}$ & $\begin{array}{r}(5) \\
(8) \\
(28) \\
(38)\end{array}$ & $\begin{array}{l}6.9 \mathrm{~b} \\
7.2 \mathrm{a} \\
7.1 \mathrm{ab} \\
6.9 \mathrm{~b}\end{array}$ & $\begin{array}{l}\text { Rom } \\
(163) \\
(200) \\
(185) \\
(163)\end{array}$ & $\begin{array}{ll}\text { Beauty-Belts } \\
7.4 \text { a } & (250) \\
7.8 \text { a } & (325) \\
7.7 \text { a } & (310) \\
7.4 \text { a } & (250)\end{array}$ & $\begin{array}{l}1.1 \mathrm{~b} \\
3.2 \mathrm{~b} \\
9.0 \mathrm{a} \\
7.7 \mathrm{a}\end{array}$ & $\begin{array}{l}4.5 \mathrm{a} \\
4.8 \mathrm{a} \\
1.8 \mathrm{a} \\
1.7 \mathrm{a}\end{array}$ & $\begin{array}{l}0.13 \mathrm{~b} \\
0.16 \mathrm{~b} \\
0.61 \mathrm{a} \\
0.74 \mathrm{a}\end{array}$ \\
\hline
\end{tabular}

break off showed bark disorders also consistent with this disease, which had been present in nearby apple orchards at Beltsville for $>10$ years.

\section{Discussion}

Although micropropagated apple trees can be produced more cheaply than budded trees, TC trees will be used in preference to budded ones only if they provide a higher net return to the grower. Net return will be affected by initial cost of trees, cost of cultural practices, and harvestable yield per hectare. Evaluation of TC trees must consider cultivars, rootstock, cultural practices employed, and orchard planting systems used, as well as climate, soils, and other site-related factors.

$\mathrm{TC}$ trees were equivalent in vigor to trees on seedling rootstock under the conditions of this experiment, although comparative vigor of these two types of root systems varied with cultivar and planting site (Fig. 1, Tables 1 and 2). As anticipated, the TC trees of standard cultivars were larger than trees budded on either MM.106 or M.26, as shown also for MM.106 by Webster et al. (1985), Rosati and Gaggioli (1987, 1989), and Cobianchi et al. (1988). The relative difference between the TC trees and those on MM. 106 or M.26 was much greater at Beltsville than at Kearneysville. At least four factors could have contributed to this difference: soil type, fertilizer rate, herbicide strip width, and nematode infestation. The soil at Kearneysville appears to be superior based on growth of other fruit trees and the sod strips in the orchard. Trees at Kearneysville received higher fertilizer rates the first 2 years and throughout the experiment had less competition for nutrients and water because of the wider herbicide-treated strip. Perhaps the most important factor between locations was nematode infestation at Beltsville, which was increasing in severity as determined by the nematode counts in succeeding years, with the resultant high incidence of apple union necrosis and decline. In this regard, the TC trees were less affected by nematodes, probably because of the more extensive root systems on the TC trees, and by tomato ring spot virus, because of the lack of a graft union.

Flowering in TC trees was delayed compared with budded trees, as reported also by Webster et al. (1985) and Rosati and Gaggioli (1987). This effect was exacerbated by root pruning in the nursery lifting operation and shoot pruning at time of planting done to equalize the size of trees. Unpruned TC trees of the same cultivars planted at Beltsville that same year (1982) flowered 1 year earlier than did the trees in this experiment. In 
subsequent studies, TC trees established as actively growing trees directly in the orchard $(\approx 5$ months after rooting in culture) began flowering as early as the third growing season and even in the second growing season in two cases (Zimmerman, 1983; Zimmerman and Miller, 1986; Zimmerman and Steffens, 1989). TC trees appear to behave like juvenile seedling trees in respect to flowering since any cultural practices that result in significant pruning of either roots or shoots or other treatments that would restrict early growth of the trees will delay flowering (Zimmerman, 1972).

Fruit yields were lower than anticipated on all trees, with the possible exception of 'Ozark Gold' on MM.106 and M.26 at Kearneysville. The experiment was terminated too early to obtain a good evaluation of fruiting, but the very poor growth of trees budded on MM.106 and M.26 at Beltsville indicated that no further useful information could be gained from this study. Webster et al. (1985), Rosati and Gaggioli (1987, 1989), and Cobianchi et al. (1988) all found that TC trees came into bearing later than did budded trees on MM.106 (the one common rootstock in all four studies) and that early yields were lower on the TC trees. However, Rosati and Gaggioli (1987) found that TC trees of the Perleberg 3 clone of spur 'Golden Delicious' had produced as much fruit after four seasons as the same clone on MM. 106, and both types had produced significantly more fruit than trees on MM.111. In the 5th year, TC trees produced significantly more fruit than trees on MM.106 or MM.111 (Rosati and Gaggioli, 1989). However, efficiency of the TC trees was lowest and that of trees on MM.106 highest because of the greater vigor of the $\mathrm{TC}$ trees.

Comparison of TC trees with budded or grafted trees is comlex because the latter type has a root system 1 year older than the top, and the root system is usually 3 years old at the time of orchard planting. In addition, the cultural practices used for preparing TC trees for field planing have varied widely (Cobianchi et al., 1988; Rosati and Gaggioli, 1987; Webster et al., 1985; Zimmerman and Steffens, 1989) and these differences have clearly affected the results obtained (Zimmerman, 1986). From these experiments, one can conclude that planting very small TC plants while dormant (Webster et al., 1985) or large ones after 2 years in a nursery, if these are heavily pruned at planting time (this study), does not provide a true evaluation of the potential of TC trees. Growing TC trees in the nursery for 1 year, as done by Rosati and Gaggioli (1985) and Cobianchi et al. (1988), fits with current commercial practice and seems not to delay fruiting unduly. Probably the most promising technique for field establishment is the use of actively growing TC plants in early June, particularly if one accepts the necessity of using a light-duty staking system for the first 2 or 3 years. This system permits vigorous vegetative growth that produces a wellbranched tree in the first year in the field (Zimmerman and Steffens, 1989). In addition, the utility of TC trees will depend greatly on the precocity of the cultivar so that extensive testing of both standard and spur types will be required before decisions on adaptability of this propagation technique can be made.

\section{Literature Cited}

Cobianchi, D., F.R. De Salvador, W. Faedi, W. Insero, A. Liverani, L. Rivalta, and A. Minguzzi. 1988. Preliminary field observations on in vitro propagated trees. Acta Hort. 227:514-516.

Hutchinson, J.F. and R.H. Zimmerman. 1987. Tissue culture of temperate fruit and nut trees. Hort. Rev. 9:273-349.

Jones, O. P., C.A. Pontikis, and M.E. Hopgood. 1979. Propagation in vitro of five apple scion cultivars. J. Hort. Sci. 54:155-158.

Korcak, R. F., R.H. Zimmerman, and S.S. Miller. 1988. Foliar nutrient status of field grown tissue cultured and budded apple trees. J. Plant Nutr. 11:1649-1662.

Larsen, F. E., S.S. Higgins, and R.H. Zimmerman. 1989. Early performance of several self-rooted apple cultivars vs. trees on seedling or M7a rootstock. ASHS 1989 Annu. Meeting, Tulsa, Okla., Prog. \& Abstr. p. 88.

Rosati, P. and D. Gaggioli. 1987. Field performance of micropropagated peach rootstock and scion cultivars of sour cherry and apple. Acta Hort. 212:379-390.

Rosati, P. and D. Gaggioli. 1989. Orchard response of micropropagated sour cherry and apple cultivars, Scientia Hort. 39:201-209.

SAS Institute, Inc. 1982. SAS user's guide: statistics. SAS Institute, Inc., Cary, N.C.

Webster, A. D., V.D. Oehl, J.E. Jackson, and O.P. Jones. 1985. The orchard establishment, growth and precocity of four micropropagated apple scion cultivars. J. Hort. Sci. 60:169-180.

Zimmerman, R.H. 1972. Juvenility and flowering in woody plants: a review. HortScience 7:447-455.

Zimmerman, R.H. 1977. Relation of pear seedling size to length of the juvenile period. J. Amer. Soc. Hort. Sci. 102:443-447.

Zimmerman, R.H. 1983. Factors affecting in vitro propagation of apple cultivars. Acta Hort. 131:171-178.

Zimmerman, R.H. 1984. Apple, p. 369-395. In: W.R. Sharp, D.A. Evans, P.V. Ammirato, and Y. Yamada (eds.). Handbook of plant cell culture, vol. 2. Crop species. Macmillan, New York.

Zimmerman, R.H. 1986. Propagation of fruit, nut, and vegetable cropsoverview, p. 183-200. In: R.H. Zimmerman, R.J. Griesbach, F.A. Hammerschlag, and R.H. Lawson (eds.). Tissue culture as a plant production system for horticultural crops. Martinus Nijhoff, Dordrecht, the Netherlands.

Zimmerman, R.H. and O.C. Broome. 1980. Apple cultivar micropropagation. Proc. Conf. Nursery Production of Fruit Plants Through Tissue Culture-Applications and Feasibility. U.S. Dept. Agr. Sci. Educ. Admin., ARR-NE-11. p. 54-58.

Zimmerman, R.H. and O.C. Broome. 1981. Phloroglucinol and in vitro rooting of apple cultivar cuttings. J. Amer. Soc. Hort. Sci. 106:648-652.

Zimmerman, R.H. and S.S. Miller. 1985. Field growth of tissue-cultured compared to budded apple trees. HortScience 20:584. (Abstr.)

Zimmerman, R.H. and S.S. Miller. 1986. Early yields from tissuecultured apple trees. HortScience 21:814. (Abstr.)

Zimmerman, R.H. and G.L. Steffens. 1989. Management of self-rooted tissue-cultured apple trees. I. Orchard establishment and early growth. Acts Hort. 239:117-120. 